\title{
Primary biphasic synovial sarcoma of the orbit
}

\author{
N Ratnatunga, J R Goodlad, N Sankarakumaran, R Seimon, S Nagendran, \\ C D M Fletcher
}

\begin{abstract}
Synovial sarcoma is one of the most common soft tissue malignancies of adolescents and young adults. Despite its name, it is no longer thought to be histogenetically derived from the synovium. What seems to be the first case of synovial sarcoma to arise in the orbit presented in a 21 year old woman as a slowly enlarging subconjunctival mass. Although this tumour was typically biphasic, the monophasic spindle cell variant arising at this site could easily be confused with less aggressive orbital connective tissue neoplasms.
\end{abstract}

Soft tissue tumours of the orbit, particularly sarcomas, are rare, ${ }^{1}$ with the notable exception of rhabdomyosarcoma (most often of the embryonal type) in infants and young children. ${ }^{2}$ In adults, the most common lesions are benign fibrous histiocytoma and haemangiopericytoma. Synovial sarcoma represents one of the most common soft tissue malignancies in adolescents and young adults and predominates in the leg, most often arising in the thigh or around the knee. Less than $10 \%$ of cases arise in the head and neck region, ${ }^{34}$ occurring most often in a paravertebral location. But the existence of these tumours, which include lesions of the oral cavity and cheek, combined with comparable lesions arising in such sites as the anterior abdominal wall and retroperitoneum, provided one of the earliest pieces of evidence that synovial sarcoma is not histogenetically derived from synovium. In fact, histogenesis is no longer regarded as tenable in malignant mesenchymal tumours generally. ${ }^{5}$ To our knowledge, no such tumour has been reported as arising in the orbit.

Department of

Histopathology, St

Thomas's Hospital

(UMDS), London

J R Goodlad

CD M Fletcher

Department of

Histopathology,

University of

Peradeniya, Sri Lanka

N Ratnatunga

N Sankarakumaran

Department of

Ophthalmology,

General Hospital,

Kandy, Sri Lanka

$R$ Seimon

$S$ Nagendran

Correspondence to:

Dr C D M Fletcher, Sof

Tissue Tumour Unit,

Department of

Histopathology, St Thomas's

Hospital, London SE1 7EH

Accepted for publication

31 July 1991 of a specific diagnosis an incomplete excision was performed (leaving a $7 \mathrm{~mm}$ portion of tumour on the posterior aspect of the globe) and the eye was preserved. The patient has

\section{Case history}

A 21 year old Sri Lankan woman presented with a five year history of a progressively enlarging, painless mass in the left orbit which had resulted in lateral displacement of the eyeball. Examination showed the presence of a $2 \mathrm{~cm}$ subconjunctival tumour on the medial side, associated with limited adduction of the left eye. There was no proptosis and vision was normal. At surgery the tumour was adherent to the epimysium of the medial rectus muscle and extended posteriorly along the medial orbital wall into the retrobulbar region. In the absence since refused further treatment. Following the definitive histological report a thorough clinical and radiological search has been made for a primary lesion elsewhere in the head and neck, trunk, and limbs with negative results.

\section{Pathological findings}

The specimen consisted of a lobulated mass of soft pale tissue measuring $3 \times 1.5 \times 1.5 \mathrm{~cm}$ with no normal surrounding tissue. Histologically it was composed of two elements. The predominant component consisted of interlacing fascicles of spindle cells with palely eosinophilic or amphophilic cytoplasm and tapering vesicular nuclei with an indistinct nucleolus (fig 1). The cells showed up to 12 mitoses per 10 high power fields $\left(1 \mathrm{hpf}=0.159 \mathrm{~mm}^{2}\right)$ and were set in a predominantly myxoid but focally hyaline stroma. Within spindle cell areas there was a branching, thin-walled vascular pattern, reminiscent of haemangiopericytoma. The other epithelioid component comprised two patterns, consisting either of solid circumscribed nests of plump epithelioid cells with

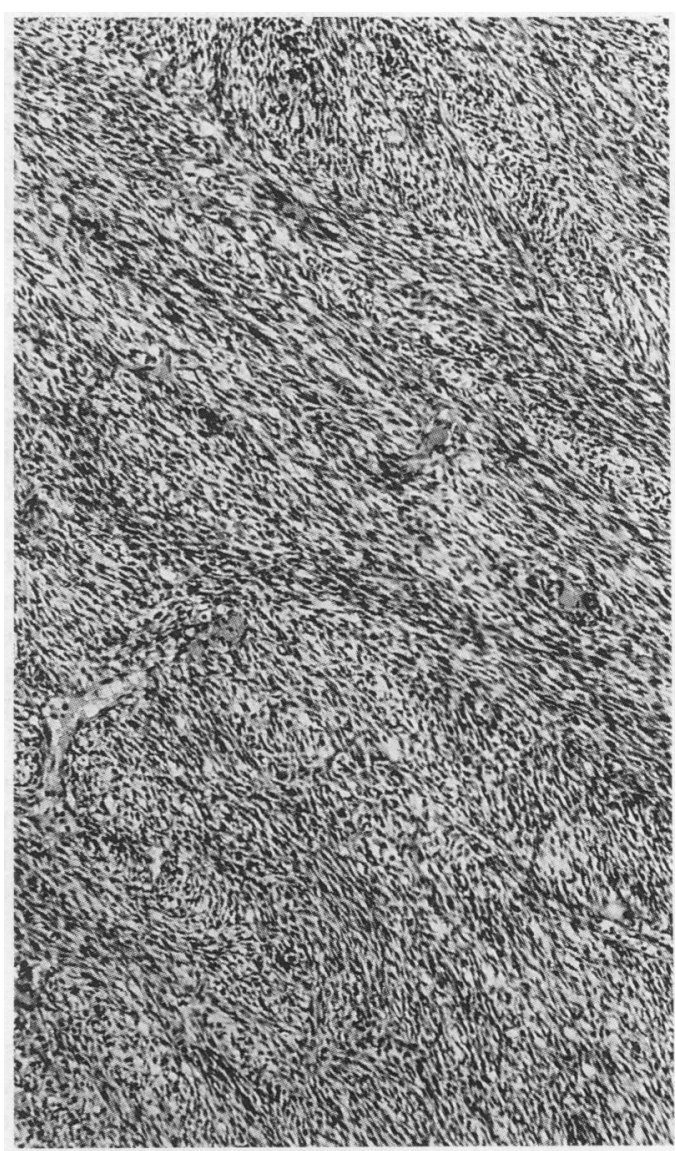

Figure 1 The spindle cell component is not easily distinguishable from a fibrosarcoma or malignant peripheral nerve sheath tumour. 

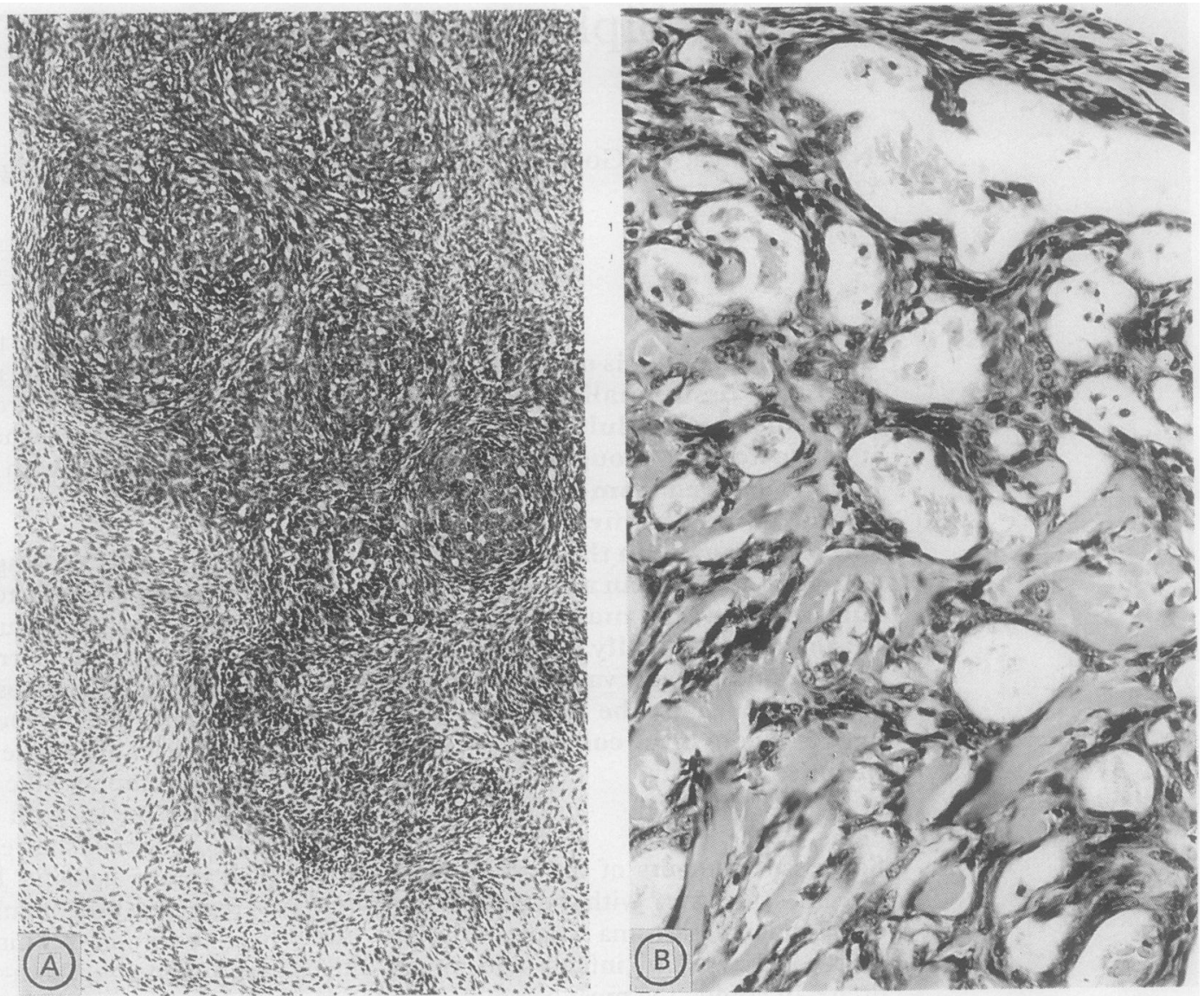

Figure 2 Areas showing epithelial differentiation formed either solid aggregates $(A)$ or glandular spaces $(B)$, many of which showed central hyalinisation.

vesicular nuclei and more eosinophilic cytoplasm, or of well formed glandular spaces arranged in small clusters (fig 2). Some of these spaces contained diastase or periodic acid Schiff positive material but most showed central hyalinisation. Both types of epithelioid area were clearly delineated by reticulin staining. In both components of the tumour, but most notably in glandular areas, there was a striking infiltrate of mast cells. At the periphery of the biopsy specimen, tumour irregularly infiltrated the medial rectus muscle.

Immunohistochemically, using the avidinbiotin complex method, both solid and glandular epithelioid elements expressed epithelial membrane antigen (EMA) (Dako) and pankeratin (DPC Ltd) and, in fact, these antibodies showed more extensive epithelial areas than were apparent in haematoxylin and eosin stained sections (fig 3). A few spindle cells also expressed EMA but were keratin negative. Stains for desmin, smooth muscle actin, S-100 protein and carcinoembryonic antigen (CEA) were negative. The appearances were typical of biphasic synovial sarcoma.

\section{Discussion}

We believe that this is the first reported example of a primary synovial sarcoma of the orbit. If this is the case it therefore expands both the range of primary sites for this tumour and the range of connective tissue tumours found in the orbit. Although this tumour was biphasic and

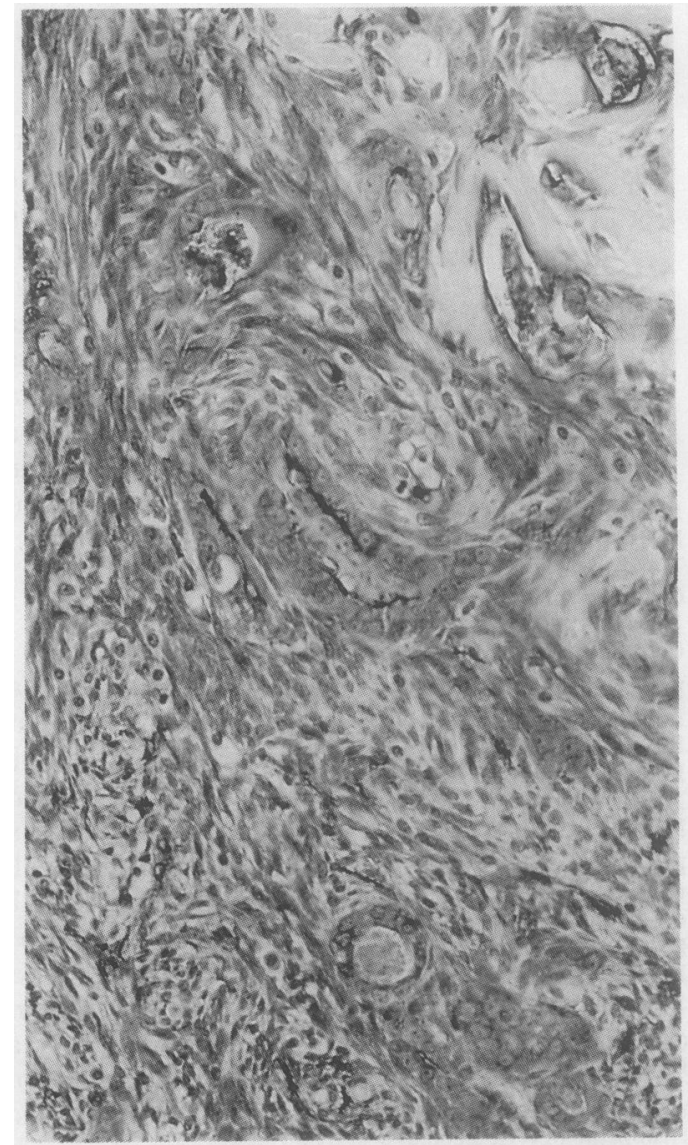

Figure 3 Glandular spaces show predominantly luminal positivity for EMA. Note also the scattered positive spindle cells ( $A B C$ method). 
therefore fairly readily recognisable, it is logical to assume that the monophasic spindle cell variant of synovial sarcoma might also present at this site and, given that such tumours often have a haemangiopericytoma-like pattern, problems in differential diagnosis might arise. Orbital haemangiopericytoma, ${ }^{6}$ which in most cases is less aggressive than synovial sarcoma, is best distinguished by its lack of EMA expression and also, less reliably, by its composition of more patternless, rounded, undifferentiated cells rather than a fascicular spindle cell appearance. Deep fibrous histiocytoma, ${ }^{7}$ well recognised in the orbit, ${ }^{8}$ is often pericytomalike, is usually only locally aggressive, and may be distinguished by its greater cytological polymorphism, a storiform architecture and EMA negativity. Overtly malignant cases show pronounced pleomorphism. Finally, infantile fibrosarcoma of the orbit ${ }^{9}$ may be both fascicular and pericytoma-like and does not usually metastasise. It can be distinguished from monophasic synovial sarcoma by its very early age of onset, EMA negativity, and by electron microscopic examination if necessary.

\section{Addendum}

Since submission of this manuscript, we have encountered a second example of primary orbital synovial sarcoma, presenting in a 42 year old Sri Lankan woman with an eight month history of left-sided orbital swelling. In this tumour, measuring about $2 \mathrm{~cm}$, which was adherent to the tendon sheath of the superior oblique muscle, the epithelial/glandular component was much less conspicuous but there was convincing EMA and keratin positivity. Such rapid identification of a second case, while possibly fortuitous, suggests that synovial sarcoma of the orbit may be underrecognised and perhaps more often misdiagnosed as haemangiopericytoma or fibrosarcoma than is commonly thought.

CDMF is a Cancer Research Campaign senior clinical research fellow.

1 Shields JA, Bakewell B, Augsburger JJ, Flanagan JC. Classification and incidence of space-occupying lesions of the orbit. A survey of 645 biopsies. Arch Ophthalmol 1984;102:1606-11.

2 Maurer HM, Beltangady M, Gehan EA, et al. The Intergroup Rhabdomyosarcoma study-1. A final report. Cancer 1988;61:209-20.

3 Roth JA, Enzinger FM, Tannenbaum M. Synovial sarcoma of the neck: a follow-up study of 24 cases. Cancer 1975;35:1243-53.

4 Shmookler BM, Enzinger FM, Brannon RB. Orofacial synovial sarcoma. A clinicopathologic study of 11 cases and review of the literature. Cancer 1982;50:269-76.

5 Fletcher CDM, McKee PH. Pathobiology of soft tissue tumours. Edinburgh: Churchill Livingstone, 1990.

6 Croxatto JO, Font RL. Hemangiopericytoma of the orbit: a clinicopathologic study of 30 cases. Hum Pathol 1982; 13:210-8.

7 Fletcher CDM. Benign fibrous histiocytoma of subcutaneous and deep soft tissue: a clinicopathologic analysis of 21 cases. Am J Surg Pathol 1990;14:801-9.

8 Font RL, Hidayat AA. Fibrous histiocytoma of the orbit. A clinicopathologic study of 150 cases. Hum Pathol 1982; 13:199-209.

9 Weiner JM, Hidayat AA. Juvenile fibrosarcoma of the orbit and eyelid. A study of five cases. Arch Ophthalmol 1983; 101:253-9.

\title{
Chicken pox infection (varicella zoster virus) and acute monoarthritis: Evidence against a direct viral mechanism
}

\author{
C G Fink, S J Read, G Giddins, R P Eglin
}

\section{Department of \\ Virology, Public \\ Health Laboratory, \\ John Radcliffe \\ Hospital, \\ Oxford OX3 9DU \\ C G Fink, S J Read, \\ R P Eglin \\ Department of \\ Orthopaedics, John \\ Radcliffe Hospital \\ G Giddins \\ Correspondence to: \\ Dr C G Fink \\ Accepted for publication \\ 27 August 1991}

\begin{abstract}
A 9 year old boy developed acute monoarthritis of the left knee concurrent with the appearance of a varicella zoster virus (VZV) rash. Repeated VZV DNA hybridisation of the cells within the synovial fluid and synovial membrane failed to show any evidence of intracellular virus. Virus was isolated from synovial fluid 24 hours after the start of clinical infection but not later. These findings suggest that the mechanism of the arthritis is not due to viral replication inside the swollen joint.
\end{abstract}

Acute arthritis is a rare complication of varicella (chicken pox). Sixteen cases have been reported, ${ }^{1}$ and in an earlier review of eight cases five involved one large joint alone. ${ }^{2}$

\section{Case report}

A 9 year old boy presented with a 24 hour history of mild papular-vesicular eruption on the trunk. This eruption developed concurrently over 24 hours with a tender, hot and swollen left knee joint which was sufficiently uncomfortable after 12 hours to inhibit weight bearing. Further crops of vesicles developed over the trunk during the next five days and the knee joint remained acutely inflamed. No vesicles were seen near to the joint. There was no history of previous injury or arthritis and a clinical diagnosis of varicella infection (VZV) was made when the child was admitted for observation and bed rest. The vesicular rash resolved over seven days and the knee joint swelling resolved over three weeks, but complete recovery of the knee joint took two months. 